\title{
Specificity and temporal dynamics of complex bacteria-sponge symbiotic interactions
}

\author{
Johannes R. Björk, ${ }^{1}$ C. Díez-Vives, ${ }^{1}$ Rafel Coma,${ }^{2}$ Marta Ribes, ${ }^{1}$ and José M. Montoya ${ }^{1,3}$ \\ ${ }^{1}$ Instituto de Ciencias del Mar, Agencia Estatal Consejo Superior de Investigaciones Científicas, \\ Passeig Maritim de la Barceloneta 37-49, 08003, Barcelona, Spain \\ ${ }^{2}$ Centre d'Estudis Avançats de Blanes, Consejo Superior de Investigaciones Cientificas (CEAB-CSIC), \\ Accés Cala Sant Francesc 14, 17300 Blanes, Spain
}

\begin{abstract}
Microbes are known to form intricate and intimate relationships with most animal and plant taxa. Microbe-host symbiotic associations are poorly explored in comparison with other species interaction networks. The current paradigm on symbiosis research stems from species-poor systems where pairwise and reciprocally specialized interactions between a single microbe and a single host that coevolve are the norm. These symbioses involving just a few species are fascinating in their own right, but more diverse and complex host-associated microbial communities are increasingly found, with new emerging questions that require new paradigms and approaches. Here we adopt an intermediate complexity approach to study the specificity, phylogenetic community structure, and temporal variability of the subset of the most abundant bacteria associated with different sponge host species with diverse eco-evolutionary characteristics. We do so by using a monthly resolved annual temporal series of host-associated and free-living bacteria. Bacteria are very abundant and diverse within marine sponges, and these symbiotic interactions are hypothesized to have a very ancient origin. We show that host-bacteria reciprocal specialization depends on the temporal scale and level of taxonomic aggregation considered. Sponge hosts with similar ecoevolutionary characteristics (e.g., volume of tissue corresponding to microbes, water filtering rates, and microbial transmission type) have similar bacterial phylogenetic community structure when looking at interactions aggregated over time. In general, sponge hosts hypothesized to form more intricate relationships with bacteria show a remarkably persistent bacterial community over time. Other hosts, however, show a large turnover similar to that observed for free-living bacterioplankton. Our study highlights the importance of exploring temporal variability in host-microbe interaction networks if we aim to determine how specific and persistent these poorly explored but extremely common interactions are.
\end{abstract}

Key words: bacterial community dynamics; ecological networks; marine sponges; microbe-host interactions; phylogenetic community structure; specialism; temporal dynamics.

\section{INTRODUCTION}

Studies on multispecies interaction networks, in particular food webs and mutualistic networks of freeliving species (e.g., plants and the insects that pollinate them), have revealed ubiquitous structural patterns of species interactions (Montoya et al. 2006, Bascompte 2009, Ings et al. 2009). Some of these patterns have challenged prevailing wisdom based on the study of isolated pairwise interactions. Reciprocal one-to-one specialization, famously illustrated by the Madagascar star orchid and the hawk moth that pollinates it, for example, is extremely rare when the overall plant-insect pollination network is examined (Joppa et al. 2009). These interaction patterns help to understand the ecological and evolutionary processes that shape multispecies communities, and have strong implications for

Manuscript received 22 March 2013; revised 15 May 2013; accepted 23 May 2013. Corresponding Editor: H. Hillebrand.

${ }^{3}$ Corresponding author. E-mail: montoya@icm.csic.es ecosystem stability, resilience, and functioning (Dunne 2006, Montoya et al. 2006, 2009, Bascompte and Jordano 2007).

What is commonly missed in the study of species interaction networks is the link between eukaryotes and prokaryotes. Although generally agreed that prokaryotes are extremely abundant, diverse, and present in all environments, that they constitute the majority of the branches in the tree of life, and that they regulate ecosystem functioning (Pedrós-Alió 2006, Fuhrman 2009), they are commonly neglected in species interaction network studies. Microbes, in general, and prokaryotes, in particular, are usually considered as a black box that interacts with almost any other species within a given ecosystem (Ings et al. 2009).

In parallel, and disconnected from species interaction network studies, microbe-host symbiotic associations have received considerable attention. There exists an enormous diversity of microbe associations with larger organisms (Moya et al. 2008), demonstrating a contin- 
uum of association strengths and types, which are often of great importance for the development, health, and functioning of the host (Robinson et al. 2010). Hostmicrobe studies are usually performed in relatively species-poor microbial systems usually characterized by extreme one-to-one or two-to-one reciprocal specializations, illustrated by the intimate associations between, e.g., Buchnera-aphids, Rhizobia-legumes, and Wolbachia-arthropods (see Ruby 2008 for a review).

One major challenge is to study multiple microbes associated with multiple hosts in more diverse systems. The associated microbiota to different parts of the human body is an example. It has shown evidence for an enormous complexity, host specificity, and temporal stability of the many dense and diverse microbial communities inhabiting the human biome (Parfrey and Knight 2012).

Here we investigate the specificity and temporal dynamics of multiple bacteria associated with different host species within a particular habitat. Our host species are marine sponges (Porifera), which represent the phylogenetically oldest, still extant metazoan phyla, with the hypothesized oldest host-bacteria symbiotic interactions (Taylor et al. 2007). Moreover, it is the group among invertebrates having the most abundant and diverse microbial community (Hentschel et al. 2012, Webster and Taylor 2012).

Sponges are commonly divided into two groups that result from the combination of a number of different ecological and evolutionary characteristics. Some (high microbial abundance) sponges harbor extremely abundant and diverse microbial communities, including species from nearly all major prokaryotic linages, while other (low microbial abundance) sponges only host a few (Hentschel et al. 2002). The observed difference in microbial community structure and complexity is hypothesized to reflect the host environment (morphology and physiology of the host), the former having a denser and more intricate structure than the latter. Although both types can form symbiotic relationships with microbes (Thacker and Starnes 2003, Schlaeppy et al. 2010), communities associated with low-abundance species are usually very similar to those found in the ambient bacterioplankton, indicating horizontally acquisition of microbes, while communities associated with high abundant species are very different (Hentschel et al. 2012, Webster and Taylor 2012). Moreover, the larvae from high-abundance species have been shown to harbor a subset of the microbial community of the adult with both vertical and horizontal transmission shown to be important in the maintenance of symbiotic associations (Schmitt et al. 2008, Lee et al. 2009, Webster et al. 2010). These two host strategies can be viewed as two different eco-evolutionary responses to their association with different symbiotic microbes. High-microbialabundance sponges tend to depend more on nutrients produced by their associated microbes, while lowmicrobial-abundance sponges tend to be highly depen- dent on nutrients taken up from the water column. This is reflected by differences in water pumping rates and sponge tissue density: high-microbial-abundance sponges have lower pumping rates and a substantially denser tissue than low-microbial-abundance hosts (Weisz et al. 2008, Ribes et al. 2012). These differences, together with other mechanisms, e.g., host defense and symbiont recognition, may suggest of two different evolutionary trajectories that have resulted in profound morphological and physiological differences.

Here we adopt an intermediate complexity approach to study host-microbe interaction networks. This means we neither focus on single host-microbe associations nor consider the entire "rare biosphere" (i.e., bacteria at very low abundances) that have been described in these systems (Hentschel et al. 2012). We study the most abundant members of the bacterial community associated with three Mediterranean sponges, and the freeliving bacterioplankton (i.e., bacteria living in surrounding seawater not associated to any host) they filter to feed. In particular, we ask three questions: (1) How specific are the most abundant bacterial species to the host they are associated within a given habitat? (2) How persistent are host-bacteria associations over time? and (3) Does host eco-evolutionary characteristics, i.e., high- and low-microbial sponges, determine bacterial specificity and persistence of the associations?

\section{Methods}

\section{Bacteria-sponge interactions}

Our study system is the Mediterranean coralligenous, a low-turnover hard-bottom community of biogenic origin produced by the accumulation of calcareous encrusting algae in dim-light conditions. The ecological relevance of this emblematic community is due to its high diversity (up to 1666 species [Ballesteros 2006]), which is a consequence of the structural complexity provided by engineering species such as calcareous algae and sponges. Sponges are ancient metazoans abundant in most hard-bottom substrata around the world. They are potential key species in benthic-pelagic coupling due to their ability to continuously clear large water volumes, particularly from picoplankton (Gili and Coma 1998).

We collected our samples close to Islas Medas, in the northwestern Mediterranean Sea $\left(42^{\circ} 3^{\prime} 0^{\prime \prime} \mathrm{N}, 3^{\circ} 13^{\prime} 0^{\prime \prime}\right.$ E). Specimens of sponges Agelas oroides, Chondrosia reniformis, and Dysidea avara were collected monthly for a year (March 2009-February 2012) by scuba-diving (depths of 5-10 m). These sponges represent a subset of the most abundant species in this sublittoral ecosystem. The two former are categorized as high-microbialabundance (HMA) species, while the latter is categorized as a low-microbial-abundance (LMA) species (Vacelet and Donadey 1977, Schlaeppy et al. 2010).

During dives, specimens were placed in separate plastic bottles and brought to the surface where we carefully transferred them to jars containing $2 \mathrm{~L}$ of 
filtered seawater $(0.22-\mu \mathrm{m}$ filter $)$. In parallel, but independent from specimen collection, samples of the ambient seawater at $5 \mathrm{~m}$ depth were taken to analyze free-living bacterioplankton. Samples were transported in an insulated cooler to the laboratory $(<2 \mathrm{~h})$ where specimens were cut into small pieces and frozen at $-80^{\circ} \mathrm{C}$ until DNA extraction. Triplicate aliquots of seawater (300-500 $\mathrm{mL}$ each) were filtered through $0.2-\mu \mathrm{m}$ polycarbonate filters, which were subsequently submerged in lysis buffer and frozen at $-80^{\circ} \mathrm{C}$.

The same individual was not sampled twice. This was done to avoid observing an effect in bacterial community composition caused by a decline in host condition. Stressed-induced individuals have been shown to change their community composition (Mohamed et al. 2008, Webster et al. 2011). In addition, intraspecific variation in host bacterial communities associated to sponges is generally small (Taylor et al. 2007, Schmitt el al. 2012).

\section{Sequence and data analysis}

Tissue samples from each sponge specimen were dissected into small sections using a sterile scalpel. Total genomic DNA was extracted from sponges and from filters using a commercial kit (DNeasy Blood and Tissue Kit; Qiagen, Hilden, Germany). Purified DNA was quantified using spectrophotometry (Nanodrop; Thermo Scientific, Waltham, Massachusetts, USA) and subsequently used as a template in the PCR. General 16S rDNA bacterial primers, GC-358F and 907RM, were used (Muyzer and Smalla 1998). Denaturing gradient gel electrophoresis (DGGE) separate the PCR amplicons ( $\sim 586$ bps), generating band fingerprints mirroring the most abundant members of the bacterial community (Muyzer et al. 1993, Fromin et al. 2002). An individual discrete band corresponds to a unique sequence type or phylotype (hereafter species), which are treated as a discrete bacterial population. The abundance of the most abundant subset of each bacterial population was estimated using band image analysis. Band intensity has been shown to be correlated to the relative abundance of the corresponding bacterial species (Fromin et al. 2002). Although, used before (Tomotada et al. 2000, Schauer et al. 2003, Pascoal et al. 2010), this approach of inferring relative abundance is debated since it has been shown to produce biased estimates (Neilson et al. 2013). This is why we use it here as a mere proxy for relative abundance. Moreover, we use it as a proxy for comparisons among samples that might be affected by the same bias. Bands within the same position in the gel in different months correspond to identical species. This allows for assessing variation on their relative abundances over time. A subset from the total number of bands, the most representative (i.e., the most intense) were excised and re-amplified with the original primer set (without the GC-clamp). The products were submitted for automated DNA sequencing (Macrogen, Amsterdam, Netherlands) for direct sequencing (Appendix A: Fig A1; Appendix C: Table
C1). DGGE images were analyzed using the QuantityOne software package (Bio-Rad, Hercules, California, USA). The contribution of individual bands was calculated relative to that of the total absorbance of each lane. Sequences were aligned against the SILVA reference database (Pruesse et al. 2007) using the kmer search option and the Needleman-Wunch algorithm. The alignment was filtered, using the default settings, to remove noninformative gaps introduced in the previous step. This was performed using the Mothur package (Schloss et al. 2009). Gene sequences were deposited in Genbank under accession numbers KC200485KC200545.

\section{Specificity and temporal dynamics of bacteria- host interactions}

To assess different dimensions of specificity and temporal dynamics of bacteria-host associations in different hosts, we used two different temporal scales. First, we used monthly resolved host-bacteria associations. This resulted in 12 different bacterial community structures for each host and for the bacterioplankton. We called these monthly resolved communities. Second, we pooled all the bacterial species present over the year in each host and in the bacterioplankton. This resulted in one community structure for each host, hereafter called the aggregated community.

At both temporal scales, we performed a number of analyses to determine differences in the level of hostbacteria specificity between different hosts and the bacterioplankton. To determine temporal dynamics we used monthly resolved communities.

Community similarity.-First, we aimed at comparing how similar bacterial communities are among them in terms of the presence/absence of bacterial species. The similarity of community membership between hosts $i$ and $j$ is defined by the standard Jaccard similarity index $J_{i j}^{\alpha}$ :

$$
J_{i j}^{\alpha}=\frac{A_{i j}^{\alpha}}{A_{i j}^{\alpha}+B_{i j}^{\alpha}+C_{i j}^{\alpha}}
$$

where $A$ is the total number of sequences present in both hosts, $B$ is the number of sequences present in $i$ but not in $j$, and $C$ is the number of sequences present in $j$ but not in $i$, and $\alpha$ is the distance cut-off to consider two sequences as the same. By changing $\alpha$ it is possible to compare community similarity at different levels of taxonomic resolution. This is important if we aim at determining whether specificity occurs at fine or broad bacterial taxonomic resolutions. Here we use individual non-overlapping DGGE bands as a proxy for species level, and an $\alpha$ of $5 \%$ and $20 \%$ to indicate genus and phylum level, respectively.

Sponge-specific bacterial clusters.-Second, we aimed at determining whether our bacterial species were specific of sponge hosts, and not widespread distributed among other hosts or free-living environments. To do so, we compared our sequenced bacterial species with 
previously sponge-derived bacterial species to determine whether these were specific to sponge hosts. This was done by assigning our sequences to already defined sponge-specific monophyletic clusters by incorporating sequences into existing phylogeny using the quick-add parsimony tool as implemented in the ARB software package (Ludwig 2004). A sponge-specific cluster is a sponge-derived group of at least three 16S rRNA gene sequences, which (1) are more similar to each other than to sequences from other, non-sponge sources, (2) are found in at least two host sponge species and/or in the same host species but from different geographic locations, and (3) cluster together irrespective of the phylogeny inference method used (Hentschel et al. 2002, updated in Simister et al. 2012).

Bacterial genetic diversity.-Third, we tested whether different hosts have different bacterial genetic diversity associated to them. This provides a finer resolution by introducing genetic information into how similar/different host-specific bacteria are. We did so for both aggregated and monthly resolved communities, testing whether bacterial genetic diversity is different from that which would result from pooling the diversity from any two host species. We used analysis of molecular variance (AMOVA; Excoffier et al. 1992). This method is widely used in population genetics, but can be extended to community ecology (Schloss 2008). It is a nonparametric analogue of ANOVA, based on a distance matrix of sequences retrieved from different communities. We used the uncorrected p-distance, which calculates the distance between pairs of sequences as the proportion of unique nucleotide positions. This distance does not correct for multiple nucleotide substitutions and should therefore be regarded as a raw distance of sequence divergence. We performed AMOVA with 100000 randomizations using the Mothur package (Schloss et al. 2009).

Bacterial phylogenetic diversity and structure in each host.-Fourth, we included phylogenetic information about bacteria to calculate bacterial phylogenetic diversity and structure to get an even finer resolution into how unique and phylogenetically diverse bacterial communities are among hosts and over time. Two phylogenies (aggregated and monthly resolved) were estimated, using RAxML (v.7.3.5), by maximum-likelihood interference and GTR+CAT approximation with 1.000 bootstraps (Stamatakis 2006). The two trees were rooted by including a sponge associated Archaeal 16S rRNA gene sequence (accession number EF529650) as an outgroup. We calculated phylogenetic structure using the unweighted UniFrac distance $U$ (Lozupone and Knight 2005). The UniFrac distance $U$ is a phylogenetic extension of the Jaccard index, attempting to capture the amount of evolution unique to one community compared to another. It does so by calculating the fraction of branch length between two communities in the phylogeny, leading exclusively to one or the other, but not to both, as follows:

$$
U=\frac{\sum_{i=1}^{N} l_{i}\left|A_{i}-B_{i}\right|}{\sum_{i=1}^{N} \max \left(A_{i}, B_{i}\right)}
$$

where $N$ is the number of nodes in the phylogenetic tree consisting of all bacterial sequences across hosts and months, $l_{i}$ is the branch length between node $i$ and its parent, and $A_{i}$ and $B_{i}$ are indicators equal to 0 or 1 as descendants of node $i$ are absent or present in communities $A$ and $B$ respectively. This method tests the hypothesis that lineages from two or more communities are undergoing equal rates of evolution. We performed the unweighted UniFrac with 10000 randomizations using the Mothur package (Schloss et al. 2009).

To calculate phylogenetic diversity across hosts and months, we used Faith's phylogenetic diversity (PD) index (Faith 1992). Faith's PD calculates the diversity in a community by summing the lengths of all those branches that are members of the corresponding minimum spanning path connecting species of the same community. It was calculated with 10000 randomizations using the Mothur package (Schloss et al. 2009).

Persistence of bacteria-host associations.-Finally, we used three measures of temporal persistence for the bacterial communities associated with each host and the bacterioplankton. The first was calculated by counting the number of months each bacterial species was associated to a particular host. Second, we measured the coefficient of variation (CV) of each DGGE band intensity over time associated with each host. Band intensity is correlated to the relative abundance of the corresponding bacterial species (Fromin et al. 2002). We also calculated the $\mathrm{CV}$ of the phylogenetic diversity (PD) of the bacterial community over time for each host and the bacterioplankton.

\section{Bacteria-bacteria association networks}

We applied the local similarity analysis (LSA; Ruan et al. 2006) on the monthly resolved data set to explore contemporaneous and time-lagged correlations between bacterial relative abundances within and between hosts and the bacterioplankton. LSA is a complement to ordinary correlation analysis since it has the ability of finding potentially time-delayed correlations between two species varying over a time series, which cannot otherwise be identified. To account for any seasonal effects on our dataset, we chose a time-lag limit for detecting correlations of three months. This technique provides a score for each pairwise correlation, with an associated $P$ value (see Ruan et al. [2006] for full details). To avoid the risk of false discovery rate (FDR) the software implements the $q$ value to adjust for multiple testing. We chose a stringency level of 0.05 for both the $P$ and the $q$ values. The analysis was permuted 1000 times. 
TABLE 1. Bacterial genetic differentiation (analyzed with analysis of molecular variance [AMOVA]) and phylogenetic diversity and structure (analyzed with UniFrac) between hosts and the bacterioplankton for the monthly resolved and aggregated bacterial communities.

\begin{tabular}{|c|c|c|c|c|c|c|c|c|c|c|}
\hline \multirow[b]{3}{*}{ Comparison } & \multicolumn{5}{|c|}{ Monthly resolved communities } & \multicolumn{5}{|c|}{ Aggregated communities } \\
\hline & \multicolumn{3}{|c|}{ AMOVA } & \multicolumn{2}{|c|}{ UniFrac distance } & \multicolumn{3}{|c|}{ AMOVA } & \multicolumn{2}{|c|}{ UniFrac distance } \\
\hline & $F_{\mathrm{s}}$ & $\mathrm{df}$ & $P$ & $U$ & $P$ & $F_{\mathrm{s}}$ & df & $P$ & $U$ & $P$ \\
\hline $\mathrm{Ao}-\mathrm{Cr}$ & 18.94 & 1,320 & $<0.0001 \dagger$ & 0.723 & $<0.0001$ & 1.38 & 1,28 & 0.14743 & 0.745 & 0.3374 \\
\hline Ao-Da & 44.47 & 1,298 & $<0.0001 \dagger$ & 0.916 & $<0.0001$ & 5.28 & 1,29 & $<0.0001 \dagger$ & 0.905 & $0.0012 \dagger$ \\
\hline Ao-Bp & 36.54 & 1,323 & $<0.0001 \dagger$ & 0.879 & $<0.0001$ & 3.76 & 1,30 & $<0.0001 \dagger$ & 0.878 & $0.0016^{\dagger}$ \\
\hline Cr-Da & 19.95 & 1,250 & $<0.0001 \dagger$ & 0.902 & $<0.0001$ & 2.57 & 1,27 & $0.00762 \dagger$ & 0.894 & $0.0013 \dagger$ \\
\hline Cr-Bp & 25.25 & 1,275 & $<0.0001 \dagger$ & 0.906 & $<0.0001$ & 2.93 & 1,28 & $0.00039 \dagger$ & 0.918 & $0.0004 \uparrow$ \\
\hline $\mathrm{Da}-\mathrm{Bp}$ & 14.98 & 1,253 & $<0.0001 \dagger$ & 0.678 & $<0.0001$ & 2.39 & 1,29 & 0.02854 & 0.697 & 0.0126 \\
\hline
\end{tabular}

Notes: Ao corresponds to A. oroides, Cr to C. reniformis, Da to D. avara, and Bp to bacterioplankton. Each $P$ value is presented with its corresponding statistic (i.e., $F_{\mathrm{s}}$ and $U$ ).

$\dagger$ Significant difference between hosts after multiple testing using Bonferoni correction $(P<0.0083)$.

\section{RESULTS}

DGGE analysis yielded a total of 149 bands (each representing a bacterial species) throughout the year, whereof $35,30,33$, and 51 were associated with host $A$. oroides, C. reniformis, D. avara, and the bacterioplankton, respectively. Of these bands, 61 were of good enough quality to sequence and of those, 16, 14, 15, and 16 were associated with host $A$. oroides, $C$. reniformis, $D$. avara, and the bacterioplankton, respectively. Each sequenced band (i.e., species) correspond to the most abundant subset of the bacterial community within each host.

Intraspecific variability within a month was very low for host-associated bacterial communities and the bacterioplankton, as reported earlier for different sponge species (Mohamed et al. 2008, Webster et al. 2011). For the bacterioplankton, replicates of the same month clustered together in a dendrogram using Jaccard similarity index (Appendix A: Fig A2). For hostassociated communities we could not perform cluster analysis using dendrograms because monthly replicates were done in a different gel than temporal dynamics. However, for example, for A. oroides, the mean CV of band intensities (i.e., relative abundance) within monthly replicates was significantly smaller $(0.36)$ than the mean CV of band intensities across the time series $(0.68$; ANOVA, $\left.F_{1,66}=4.051, P=0.048\right)$. Importantly, although some small qualitative (presence/absence) differences among individuals within a month were observed, the relative abundance of the species responsible for these differences was very small. In all cases, these species lay within the $33 \%$ of the least abundant species, suggesting low intraspecific variability across hosts and the bacterioplankton.

\section{Specificity and persistence of bacteria-host interactions}

We did not find any overlap on bacterial species composition between hosts and the bacterioplankton, indicating an extreme reciprocal specialization at the bacterial species level (individual DGGE bands). This was consistent at both temporal scales considered, i.e., aggregated (Table 1, Fig. 1) and monthly resolved (Table 1). When assigning sequences to genus level (i.e., by grouping sequences at a $5 \%$ distance) in the aggregated community, hosts $A$. oroides and $C$. reniformis shared $4 \%$ of sequences and D. avara shared $13 \%$ with the bacterioplankton. Increasing the level of taxonomic aggregation (e.g., at the phylum level) did not change the results qualitatively (Appendix B: Table B1). However, monthly resolved communities only showed overlap for distance cut-offs above phylum level, with bacterial communities across months showing a very high similarity within each host (Table 1, Fig. 2a).

In addition, we found that the aggregated community associated with the two HMA hosts (i.e., A. oroides and C. reniformis) could more frequently be assigned to predefined sponge-specific monophyletic clusters (Simister et al. 2012), than could the aggregated community associated with the LMA host and the bacterioplankton (Pearson chi-square test, $\chi_{3}^{2}=38.17, N_{\mathrm{Ao}}=14, N_{\mathrm{Cr}}=11$, $N_{\text {Da }}=2, N_{\text {Bp }}=0, P<0.001$ (Appendix C: Table C1). By definition, bacterial species assigned to these clusters are more closely related to each other than to bacterial species from non-sponge sources (Hentschel et al. 2002).

Similarly, bacterial genetic differentiation was very different between hosts and between hosts and bacterioplankton, when monthly resolved communities were analyzed (Table 1). Only the aggregated community of both HMA hosts (AMOVA Fs (A,28 $_{1,38}=1 . P=0.147$ ) and the LMA host and the bacterioplankton (AMOVA $\left.\mathrm{Fs}_{1,29}=2.387, P=0.028\right)$ showed similar levels of bacterial genetic differentiation.

Results from the phylogenetic community structure point in the same direction. The UniFrac analysis of the aggregated community showed a strong similarity between bacterial communities associated with the HMA hosts and between bacterial communities associated with the LMA host and the bacterioplankton (Table 1). The monthly resolved communities showed higher similarities within than between communities associated with hosts and the bacterioplankton. Fig. 2b illustrates this, with bacterial communities clustering by 


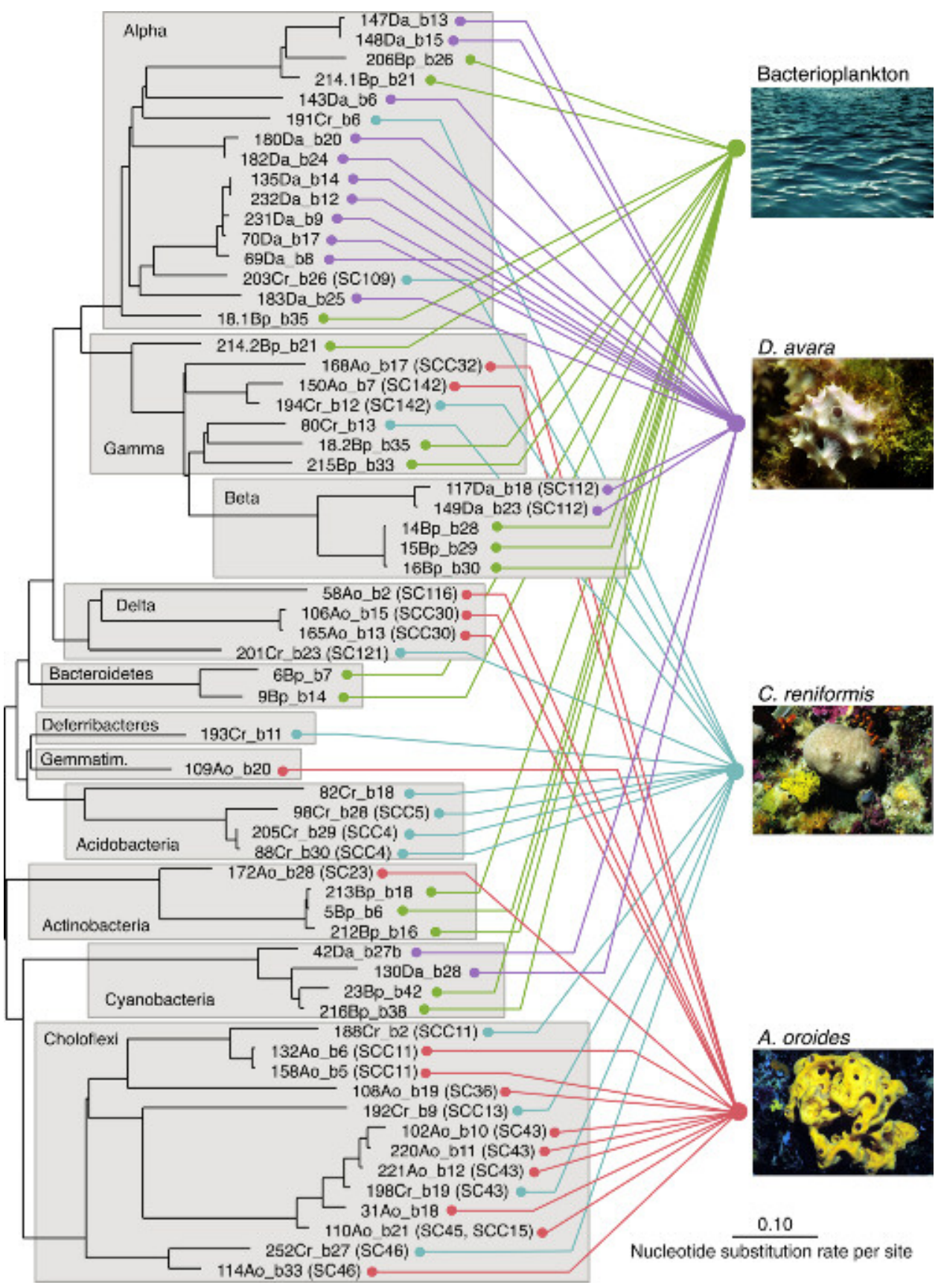

FIG. 1. Bacteria-host interaction network for the aggregated community. Each sponge host is represented as a node (to the right) with connecting edges to nodes (to the left) corresponding to bacterial sequences in a phylogeny. Each gray box corresponds to a different bacterial phylum. The abbreviation SC/SCC indicates sequences belonging to sponge-/sponge-coral-specific clusters, respectively (see Methods: Sponge-specific bacterial clusters for details; Table 2; Appendix C: Table C1). Green nodes/edges correspond to sequences found in the bacterioplankton (Bp), purple to Dysidea avara (Da), teal to Chondrosia reniformis (Cr), and red to Agelas oroides (Ao). Each bacterial species corresponds to an individual excised and sequenced denaturing gradient gel electrophoresis (DGGE) band.

host identity, with no overlap between either HMA hosts or LMA host and bacterioplankton.

However, we found support for the HMA-LMA classification of sponges when we analyzed the phylogenetic diversity of bacterial communities at both temporal scales. We found the highest diversity in the HMA hosts $A$. oroides (aggregated PD $=3.67$, monthly resolved $\mathrm{PD}=3.45$ ) and $C$. reniformis (aggregated $\mathrm{PD}=$ 4.04, monthly resolved $\mathrm{PD}=3.14$ ) and the lowest diversity in LMA host D. avara (aggregated PD $=2.29$, 

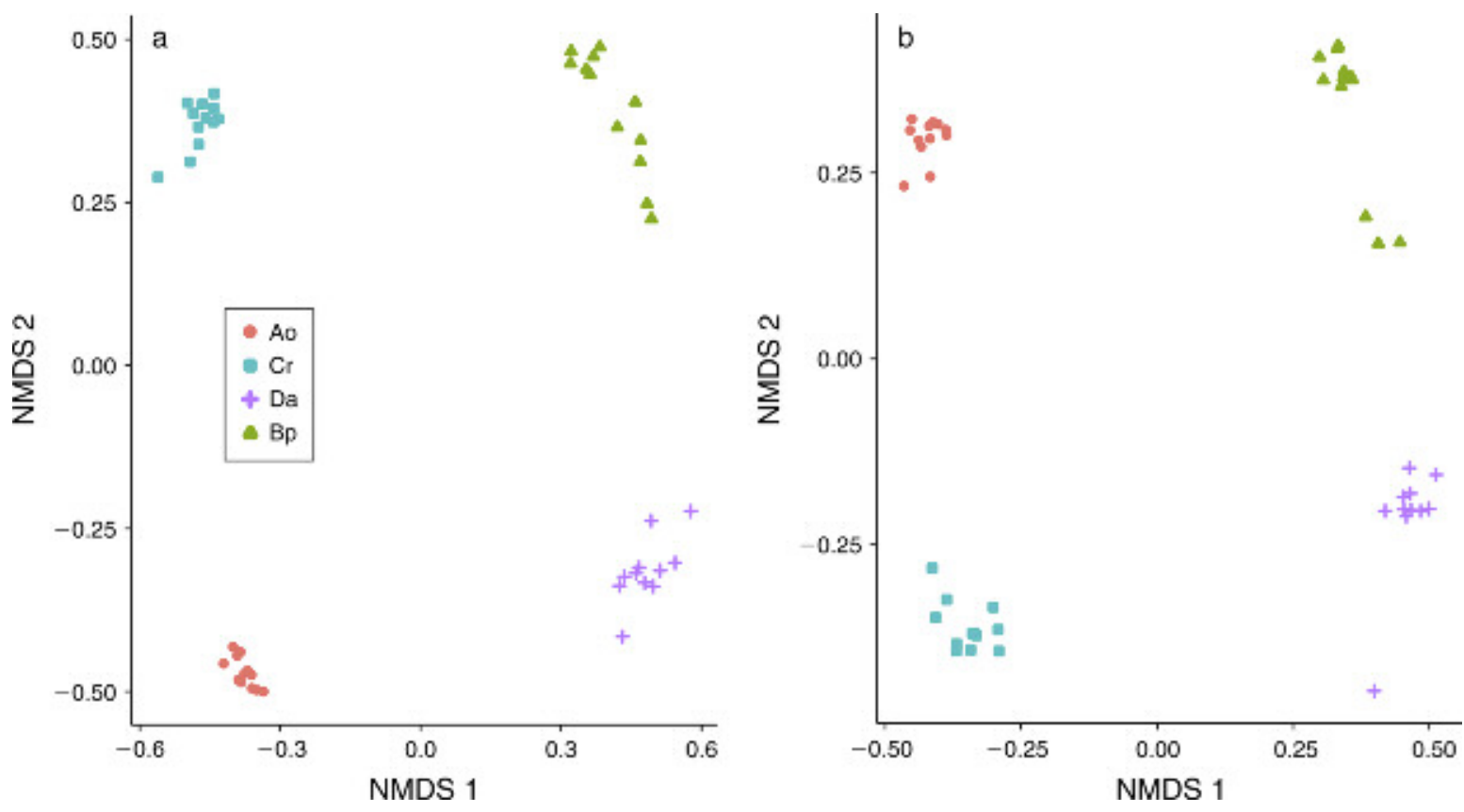

FIG. 2. (a) Nonmetric multidimensional scaling (NMDS) plot for the Jaccard index clustering species at a distance $\alpha=5 \%$, corresponding to genus level, for monthly resolved bacterial communities; lowest stress, $0.163 ; r^{2}, 0.894$. (b) NMDS plot for the UniFrac distance for monthly resolved bacterial communities; lowest stress, $0.144 ; r^{2}, 0.919$. Red circles correspond to A. oroides (Ao), teal squares to C. reniformis $(\mathrm{Cr})$, purple crosses to D. avara (Da), and green triangles to the bacterioplankton $(\mathrm{Bp})$.

monthly resolved $\mathrm{PD}=1.98$ ). Interestingly, but not surprisingly, the bacterioplankton showed a similar diversity as to the HMA hosts (aggregated PD $=3.68$, monthly resolved $\mathrm{PD}=3.14)$.

Temporal persistence of host-bacteria interactions, shown as monthly recurrrences of bacterial species within each host, was significantly higher for $A$. oroides $(9.86 \pm 0.56$ [mean $\pm \mathrm{SE}])$ and $C$. reniformis $(8.27 \pm$ $0.78)$ compared to $D$. avara $(5.76 \pm 0.62)$ and the bacterioplankton (5.71 \pm 0.52; Table 2; Fig. 3a). We further divided bacterial species into two categories according to the number of months they appeared. Permanent species were defined as those always present or only absent three months or less and temporary species were defined as those absent for three months or more. Percentages of permanent species varied across hosts, from $74 \%$ for $A$. oroides to $20 \%$ for bacterioplankton. In all hosts and bacterioplankton, permanent species contributed disproportionately more to their total bacterial abundance, while temporary species contributed disproportionately less to bacterial abundance (Table 3). For example, temporary species in $C$. reniformis (50\% of observed species) only contributed to $17 \pm 5$ per cent of total abundance. Temporary species then tend to be rare among the most abundant members of the microbial community.

Similarly, the average coefficient of variation for species relative abundances was lower for $A$. oroides (mean $\mathrm{CV}=0.27$ ) and $C$. reniformis (mean $\mathrm{CV}=0.31$ ) compared to $D$. avara (mean $\mathrm{CV}=0.41$ ) and the bacterioplankton (mean $\mathrm{CV}=0.36$ ). (Table 3; Fig. 3b). However, the observed difference between $C$. reniformis and the bacterioplankton was not significant. Also, the coefficient of variation for the phylogenetic diversity across months was lower for bacterial communities associated with $A$. oroides $(\mathrm{CV}=0.04)$ and $C$. reniformis

TABLE 2. Permanent (only absent three months or less) and temporary (absent more than three months) bacterial species and their relative contribution to total bacterial abundance (mean $\pm \mathrm{SD}$ ).

\begin{tabular}{lccccc}
\hline \hline \multicolumn{1}{c}{ Species } & $\begin{array}{c}\text { Number } \\
\text { of species }\end{array}$ & $\begin{array}{c}\text { Permanent } \\
\text { species (\%) }\end{array}$ & $\begin{array}{c}\text { Contribution of permanent } \\
\text { species to abundance (\%) }\end{array}$ & $\begin{array}{c}\text { Temporary } \\
\text { species (\%) }\end{array}$ & $\begin{array}{c}\text { Contribution of temporary } \\
\text { species to abundance (\%) }\end{array}$ \\
\hline Agelas oroides & 35 & 74 & $93 \pm 3$ & 26 & $7 \pm 3$ \\
Chondrosia reniformis & 30 & 50 & $83 \pm 5$ & 50 & $17 \pm 5$ \\
Dysidea avara & 33 & 30 & $41 \pm 12$ & 70 & $29 \pm 12$ \\
Bacterioplankton & 51 & 20 & $48 \pm 6$ & $52 \pm 6$ \\
\hline
\end{tabular}

Note: Number of species corresponds to the total number of denaturing gradient gel electrophoresis (DGGE) bands for each sponge host and the bacterioplankton. 
$(\mathrm{CV}=0.06)$ than for D. avara $(\mathrm{CV}=0.12)$ and the bacterioplankton $(\mathrm{CV}=0.20)$. Overall, we found that communities associated with the HMA hosts had a higher temporal persistence than did communities associated with the LMA host and the bacterioplankton.

\section{Bacteria-bacteria interaction networks}

Local similarity analysis (LSA) for the 12 months only revealed 12 significant correlations ( $P$ and $q$ values $\leq 0.05$ ) out of the 1830 pairwise possible correlations for the sequenced species across all hosts and bacterioplankton. This represents $0.67 \%$ when all interactions are considered, which is a much lower percentage than previously reported percentages from LSA studies from bacterioplankton communities, typically showing around $15 \%$ of correlated bacterial abundances (Ruan et al. 2006). This indicates bacterial relative abundances within hosts change more independently from each other than what is observed in free-living bacterioplankton.

\section{Discussion}

Multispecies host-bacteria symbiotic associations are poorly explored in comparison with other species interaction networks. The current paradigm on symbiosis research stems from species-poor system studies where pairwise and reciprocally specialized interactions between a single microbe and a single host that coevolve are the norm (Ruby 2008). These symbioses involving just a few species are fascinating in their own right, but more diverse and complex host-associated microbial communities are increasingly found (Robinson et al. 2010), with new emerging questions that require new paradigms and approaches. Here we have tried to answer some of these questions related to the specificity and temporal variability of the most abundant bacteria associated to different coexisting host species with diverse eco-evolutionary characteristics.

We showed that host-microbe specificity depends on both the level of microbial taxonomic aggregation and the time-scale considered. Without any taxonomic aggregation, specificity is extreme among the most abundant host-associated bacteria. Reciprocal one-toone specialization is the norm, in marked contrast to what is found in host-parasitoid systems and plantpollination networks (Joppa et al. 2009), although, in both network types, rare species are also considered. This host idiosyncratic effect dissipates when microbes are clustered at different levels of taxonomic aggregation. This is because when relaxing taxonomic aggregation and going up in taxonomic rank, one is broadening the symbionts' host-range. We found that hosts with similar eco-evolutionary characteristics share more microbes among them than with hosts with different characteristics. In particular, our two high-microbial abundant sponge species were more similar in phylogenetic community structure, while the low-microbial abundant sponge and the free-living bacterioplankton shared more phylogenetically similar microbes. This suggestive pattern needs to be confirmed in future studies using different host sponges.

Although our study focused on sponges as hosts, their eco-evolutionary characteristics allow for extrapolations to other complex microbe-host interactions. High- and low-microbial abundant sponges generally reflect differences in a number of morphological and physiological characteristics (e.g., water pumping rates, sponge tissue density) and in a number of factors that affect hostmicrobe interactions (e.g., host defense, symbiont recognition, and transmission mechanisms). This perspective of different eco-evolutionary characteristics affecting host-microbe interactions could be extended to other host multi-species systems where related hosts differ in relation to microbe acquisition/transmission and/or feeding ecology. These include, for example, complex gut microbial communities of mammals, termites, beetles, Lepidoptera, and coral-associated microbes (see Robinson et al. 2010 for a review).

The different temporal dynamics exhibited by different host species and the free-living bacterioplankton highlights the importance of considering temporally resolved interaction networks (Olesen et al. 2008, Ings et al. 2009, Lurgi et al. 2012). Otherwise, we might not capture the true differences in phylogenetic community structure. We only observed similarities in phylogenetic community structure across hosts when we aggregated bacterial communities over time. However, when timeresolved (monthly, in our case) communities were analyzed independently, no differences were observed, and bacterial communities clustered by host species. This is due to the stability of bacterial communities associated with HMA sponges: in some cases communities belonging to different months have the same phylogenetic structure, acting almost as replicates, and thus clustering together. This stability, combined with the higher temporal variability of bacterioplankton and LMA sponges, makes difficult interpreting patterns using single-time snapshot interaction networks, because this snapshot would be a good reflection of stable microbial communities but a poor reflection of continuously changing host-associated microbiota. Caution must be exercised when studying any interaction network involving species with high turnover rates. In particular, future network studies incorporating microbes would greatly benefit from adopting a temporal perspective.

This temporal perspective would allow inferring possible bacteria-bacteria associations. In contrast to hosts containing a single symbiont, in hosts containing a complex microbiota, microbe-microbe interactions can have a strong influence on the resulting microbial community (McFall-Ngai 2008, Robinson et al. 2010). However, microbe-microbe interactions are very difficult to observe directly in comparison to e.g., predatorprey interactions, and indirect methods need to be used. Temporal series analyses of relative abundances are 

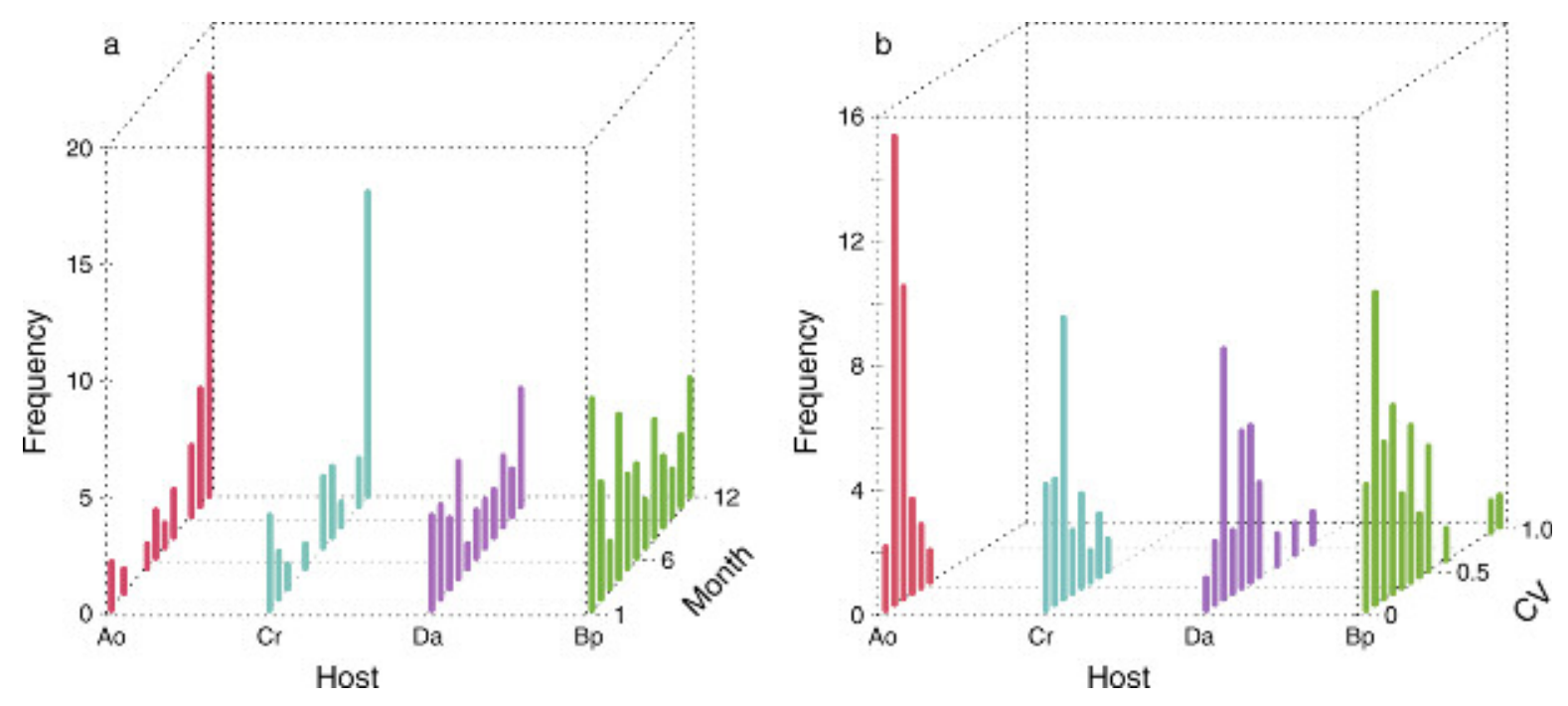

FIG. 3. Temporal persistence of bacteria-host associations, shown as (a) monthly recurrrences of bacterial species within each host (i.e., the number of months a bacterial species is present), and (b) coefficient of variation (CV) of species relative abundances. Red corresponds to A. oroides (Ao), teal to C. reniformis (Cr), purple to D. avara (Da), and green to the bacterioplankton (Bp).

useful here. We used local similarity analyses, finding that bacterial relative abundances within our hosts tend to be uncorrelated among them, in contrast to previous findings on free-living microbes (Ruan et al. 2006, Fuhrman 2009). Other methods can be used in combination to local similarity analyses to determine the importance of microbial interspecific interactions in shaping community structure, like the one developed in Mutshinda et al. (2009) to deconstruct temporal fluctuations in species relative abundances into contributions from environmental stochasticity and inter-/ intraspecific interactions. Future studies might greatly benefit from including environmental variables to disentangle its relative role in regulating bacterial dynamics.

We used DGGE band intensities as a proxy for species relative abundance, in agreement with other studies (Tomotada et al. 2000, Schauer et al. 2003,
Pascoal et al. 2010). However, caution must be exercised when interpreting relative abundances from any PCRbased method, because it can produce biased estimates of quantitative diversity measures (Neilson et al. 2013). For comparative (i.e., temporal, across host species) studies as the one presented here, these biases might affect all samples in a similar way, so changes on band intensities are likely to reflect real changes. Moreover, it is important that qualitative (based on presence/ absence) and quantitative (using relative abundance proxies) results point in the same direction.

The inclusion of phylogenetic information in network studies is increasingly recognized as a necessary step toward understanding interaction patterns and the ecoevolutionary mechanisms shaping them (Bascompte and Jordano 2007). In our case the use of molecular phylogenies based on 16S rDNA bacterial sequences allowed us for a fine-grained characterization of

TABLE 3. Temporal persistence of bacteria-host interactions, shown as monthly recurrrences of bacterial species within each host and coefficient of variation (CV), using DGGE intensities as a proxy for bacterial species relative abundances.

\begin{tabular}{llcrcr}
\hline \hline \multirow{2}{*}{$\begin{array}{c}\text { Pairwise } \\
\text { comparison }\end{array}$} & \multicolumn{2}{c}{ Monthly recurrrences } & & \multicolumn{2}{c}{ CV } \\
\cline { 2 - 3 } \cline { 5 - 5 } & \multicolumn{1}{c}{$D$} & & & $D$ & $P$ \\
\hline Ao-Cr & 0.243 & 0.296 & 0.268 & 0.200 \\
Ao-Da & 0.531 & $0.0001^{*}$ & & 0.533 & $0.001^{*}$ \\
Ao-Bp & 0.55 & $<0.001^{*}$ & 0.372 & $0.008^{*}$ \\
Cr-Da & 0.433 & $0.006^{*}$ & 0.362 & $0.040^{*}$ \\
Cr-Bp & 0.340 & $0.023^{*}$ & 0.225 & 0.328 \\
Da-Bp & 0.098 & 0.991 & 0.301 & 0.068 \\
\hline
\end{tabular}

Notes: A Kolmogorov-Smirnov test was performed for pairwise comparisons of the different distributions belonging to each host and the bacterioplankton. Each $P$ value is presented with the Kolmogorov-Smirnov statistic $D$.

$$
* P<0.05 \text {. }
$$


phylogenetic community structure. We chose an intermediate level of network complexity, focusing on the most abundant bacterial species. As massive sequencing technologies are increasingly available to characterize both the abundant and rare biosphere, we ask whether our results would hold when less abundant microbes are considered. As in other network studies, food webs in particular, focusing on intermediate complexity levels (food web modules) allows for understanding and predicting system dynamics before addressing the structure and dynamics of the broader food web (McCann 2012). The ultimate goal is to introduce microbes into ecological network studies and a network perspective into host-microbe interactions, and here we suggest as a starting point restricting the analyses to the most abundant and likely important microbes for host functioning.

\section{ACKNOWLEDGMENTS}

We thank Eduard Serrano for helping with sampling, $\mathrm{P}$ Lopez-Sendino for helping with the DNA extractions, and Lucas Joppa and an anonymous reviewer for constructive comments on the manuscript. This research was supported by a Ramon y Cajal Fellowship (RYC-892 2008-03664) to J. M. Montoya and grants from the Spanish Government to J. M. Montoya (CGL2010-20091) and R. Coma and M. Ribes (CGL2010-18466), and by a grant from the Catalan Government (2009SGR142). J. R. Björk was supported by an FPI Fellowship from the Spanish Government (BES-2011049043). J. R. Björk and C. Díez-Vives contributed equally to this work.

\section{Literature Cited}

Ballesteros, E. 2006. Mediterranean coralligenous assemblages: a synthesis of present knowledge. Oceanography and Marine Biology: An Annual Review 44:123-195.

Bascompte, J. 2009. Disentangling the web of life. Science 325: 416-419.

Bascompte, J., and P. Jordano. 2007. Plant-animal mutualistic networks: the architecture of biodiversity. Annual Review of Ecology, Evolution, and Systematics 38:567-593.

Dunne, J. A. 2006. The network structure of food webs. Pages 27-86 in M. Pascual and J. A. Dunne, editors. Ecological networks: linking structure to dynamics in food webs. Oxford University Press, Oxford, UK.

Excoffier, L., P. E. Smouse, and J. M. Quattro. 1992. Analysis of molecular variance inferred from metric distances among DNA haplotypes: application to human mitochondrial DNA restriction data. Genetics Society of America 131:479-491.

Faith, D. P. 1992. Conservation evaluation and phylogenetic diversity. Biological Conservation 61:1-10.

Fromin, N., J. Hamelin, S. Tarnawski, D. Roesti, K. JourdainMiserez, N. Forestier, S. Teyssier-Cuvelle, F. Gillet, M. Aragno, and P. Rossi. 2002. Statistical analysis of denaturing gel electrophoresis (DGE) fingerprinting patterns. Environmental Microbiology 4:634-643.

Fuhrman, J. A. 2009. Microbial community structure and its functional implications. Nature 459:193-199.

Gili, J. M., and R. Coma. 1998. Benthic suspension feeders: their paramount role in littoral marine food webs. Trends in Ecology and Evolution 13:316-321.

Hentschel, U., J. Hopke, M. Horn, A. B. Friedrich, M. Wagner, J. Hacker, and B. S. Moore. 2002. Molecular evidence for a uniform microbial community in sponges from different oceans. Applied and Environmental Microbiology 68:44314440 .
Hentschel, U., J. Piel, S. M. Degnan, and M. W. Taylor. 2012. Genomic insights into the marine sponge microbiome. Nature Reviews Microbiology 10:641-654.

Ings, T. C., et al. 2009. Review: Ecological networks - beyond food webs. Journal of Animal Ecology 78:253-269.

Joppa, L. N., J. Bascompte, J. M. Montoya, R. V. Solé, J. Sanderson, and S. L. Pimm. 2009. Reciprocal specialization in ecological networks. Ecology Letters 12:961-969.

Lee, O. O., P. Y. Chui, Y. H. Wong, J. R. Pawlik, and P.-Y. Qian. 2009. Evidence for vertical transmission of bacterial symbionts from adult to embryo in the Caribbean sponge Svenzea zeai. Applied and Environmental Microbiology 75: $6147-6156$

Lozupone, C., and R. Knight. 2005. UniFrac: a new phylogenetic method for comparing microbial communities. Applied and Environmental Microbiology 71:8228-8235.

Ludwig, W. 2004. ARB: a software environment for sequence data. Nucleic Acids Research 32:1363-1371.

Lurgi, M., B. C. López, and J. M. Montoya. 2012. Climate change impacts on body size and food web structure on mountain ecosystems. Philosophical Transactions of the Royal Society B 367:3050-3057.

McCann, K. S. 2012. Food webs. Princeton University Press, Princeton, New Jersey, USA.

McFall-Ngai, M. 2008. Are biologists in "future shock?" Symbiosis integrates biology across domains. Nature 6:789792.

Mohamed, N. M., V. Rao, M. T. Hamann, M. Kelly, and R. T. Hill. 2008. Monitoring bacterial diversity of the marine sponge Ircinia strobilina upon transfer into aquaculture. Applied and Environmental Microbiology 74:4133-4143.

Montoya, J. M., S. L. Pimm, and R. V. Solé. 2006. Ecological networks and their fragility. Nature 442:259-264.

Montoya, J. M., G. Woodward, M. C. Emmerson, and R. V. Solé. 2009. Press perturbations and indirect effects in real food webs. Ecology 90:2426-2433.

Moya, A., J. Peretó, R. Gil, and A. Latorre. 2008. Learning how to live together: genomic insights into prokaryoteanimal symbioses. Nature Reviews Genetics 9:218-229.

Mutshinda, C. M., R. B. O'Hara, and I. P. Woiwod. 2009. What drives community dynamics? Proceedings of the Royal Society B 276:2923-2929.

Muyzer, G., E. C. de Waal, and A. G. Uitterlinden. 1993. Profiling of complex microbial populations by denaturing gradient gel electrophoresis analysis of polymerase chain reaction-amplified genes coding for $16 \mathrm{~S}$ rRNA. Applied and Environmental Microbiology 59:695-700.

Muyzer, G., and K. Smalla. 1998. Application of denaturing gradient gel electrophoresis (DGGE) and temperature gradient gel electrophoresis (TGGE) in microbial ecology. Antonie van Leeuwenhoek 71:127-141.

Neilson, J. W., F. L. Jordan, and R. M. Maier. 2013. Analysis of artifacts suggest DGGE should not be used for quantitative diversity analysis. Journal of Microbiological Methods 92:256-263.

Olesen, J. M., J. Bascompte, H. Elberling, and P. Jordano. 2008. Temporal dynamics in a pollination network. Ecology 89:1573-1582.

Parfrey, L. W., and R. Knight. 2012. Spatial and temporal variability of the human microbiota. Clinical Microbiology and Infection 18:5-7.

Pascoal, C., F. Cássio, L. Nikolcheva, and F. Bärlocher. 2010. Realized fungal diversity increases functional stability of leaf decomposition under zinc stress. Microbial Ecology 59:8493.

Pedrós-Alió, C. 2006. Marine microbial diversity: can it be determined? Trends in Microbiology 14:257-263.

Pruesse, E., C. Quast, K. Knittel, B. M. Fuchs, W. Ludwig, J. Peplies, and F. O. Glockner. 2007. SILVA: a comprehensive online resource for quality checked and aligned ribosomal 
RNA sequence data compatible with ARB. Nucleic Acids Research 35:7188-7196.

Ribes, M., E. Jiménez, G. Yahel, P. López-Sendino, B. Diez, R. Massana, J. H. Sharp, and R. Coma. 2012. Functional convergence of microbes associated with temperate marine sponges. Environmental Microbiology 14:1224-1239.

Robinson, C. J., B. J. M. Bohannan, and V. B. Young. 2010. From structure to function: the ecology of host-associated microbial communities. Microbiology and Molecular Biology Reviews 74:453-476.

Ruan, Q., D. Dutta, M. S. Schwalbach, J. A. Steele, J. A. Fuhrman, and F. Sun. 2006. Local similarity analysis reveals unique associations among marine bacterioplankton species and environmental factors. Bioinformatics 22:2532-2538.

Ruby, E. G. 2008. Symbiotic conversations are revealed under genetic interrogation. Nature Reviews Microbiology 6:752762.

Schauer, M., V. Balagué, C. Pedrós-Alió, and R. Massana. 2003. Seasonal changes in the taxonomic composition of bacterioplankton in a coastal oligotrophic system. Aquatic Microbial Ecology 31:163-174.

Schlaeppy, M. L., S. I. Schottner, G. Lavi, M. M. M. Kuypers, D. de Beer, and F. Hoffmann. 2010. Evidence of nitrification and denitrification in high and low microbial abundance sponges. Marine Biology 157:593-602.

Schloss, P. D. 2008. Evaluating different approaches that test whether microbial communities have the same structure. ISME Journal 2:265-275.

Schloss, P. D., et al. 2009. Introducing mothur: open-source, platform-independent, community-supported software for describing and comparing microbial communities. Applied and Environmental Microbiology 75:7537-7541.

Schmitt, S., H. Angermeier, R. Schiller, N. Lindquist, and U. Hentschel. 2008. Molecular microbial diversity survey of sponge reproductive stages and mechanistic insights into vertical transmission of microbial symbionts. Applied and Environmental Microbiology 74:7694-7708.
Simister, R. L., P. Deines, E. S. Botté, N. S. Webster, and M. W. Taylor. 2012. Sponge-specific clusters revisited: a comprehensive phylogeny of sponge-associated microorganisms. Environmental Microbiology 14:517-524.

Stamatakis, A. 2006. RAxML-VI-HPC: maximum likelihoodbased phylogenetic analyses with thousands of taxa and mixed models. Bioinformatics 22:2688-2690.

Taylor, M. W., R. Radax, D. Steger, and M. Wagner. 2007. Sponge-associated microorganisms: evolution, ecology, and biotechnological potential. Microbiology and Molecular Biology Reviews 71:295-347.

Thacker, R. W., and S. Starnes. 2003. Host specificity of the symbiotic cyanobacterium Oscillatoria spongeliae in marine sponges, Dysidea spp. Marine Biology 142:643-648.

Tomotada, I., K. Tani, K. Nakamura, Y. Suzuki, M. Kitagawa, M. Eguchi, and M. Nasu. 2000. Monitoring impact of in situ biostimulation treatment on groundwater bacterial community DGGE. FEMS Microbiology Ecology 32:129-141.

Vacelet, J., and C. Donadey. 1977. Electron-microscope study of association between some sponges and bacteria. Journal of Experimental Marine Biology and Ecology 30:301-314.

Webster, N. S., R. E. Cobb, R. Soo, S. L. Anthony, C. N. Battershill, S. Whalan, and E. Evans-Illidge. 2011. Bacterial community dynamics in the marine sponge Rhopaloeides odorabile under in situ and ex situ cultivation. Marine Biotechnology 13:296-304.

Webster, N. S., and M. W. Taylor. 2012. Marine sponges and their microbial symbionts: love and other relationships. Environmental Microbiology 14:335-346.

Webster, N. S., M. W. Taylor, F. Behnam, S. Luecker, T. Rattei, S. Whalan, M. Horn, and M. Wagner. 2010. Deep sequencing reveals exceptional diversity and modes of transmission for bacterial sponge symbionts. Environmental Microbiology 12:2070-2082.

Weisz, J. B., N. Lindquist, and C. S. Martens. 2008. Do associated microbial abundances impact marine demosponge pumping rates and tissue densities? Oecologia 155:367-376.

\section{Supplemental Material}

\section{Appendix A}

Figures showing different analyses conducted using denaturing gradient gel electrophoresis (DGGE) analysis and a dendrogram of the monthly Jaccard coefficient of similarity for the bacterioplankton (Ecological Archives E094-257-A1).

\section{Appendix B}

A table showing the Jaccard index for different distance cutoffs, corresponding to different taxonomic levels (Ecological Archives E094-257-A2).

\section{Appendix C}

A table summarizing information about excised and sequenced DGGE bands, including GeneBank accession numbers, taxonomic affiliation, and membership to sponge-specific clusters (Ecological Archives E094-257-A3). 